\title{
Kantowski-Sachs Tsallis holographic dark energy model with sign-changeable interaction
}

\author{
Y. Sobhanbabu ${ }^{1, a}$, M. Vijaya Santhi ${ }^{2}$ \\ ${ }^{1}$ Department of Engineering Mathematics and Humanities, Sagi Rama Krishnam Raju Engineering College, Bhimavaram 534204, India \\ 2 Department of Applied Mathematics, Andhra University, Visakhapatnam 530003, India
}

\begin{abstract}
In this work devoted to the investigation of the Tsallis holographic dark energy (IR cut-off is Hubble radius) in homogeneous and anisotropic Kantowski-Sachs Universe within the frame-work of Saez-Ballester scalar tensor theory of gravitation. We have constructed non-interacting and interacting Tsallis holographic dark energy models by solving the field equations using the relationship between the metric potentials. This relation leads to a viable deceleration parameter model which exhibits a transition of the Universe from deceleration to acceleration. In interacting case, we focus on sign-changeable interaction between Tsallis holographic dark energy and dark matter. The dynamical parameters like equation of state parameter, energy densities of Tsallis holographic dark energy and dark matter, deceleration parameter, and statefinder parameters of the models are explained through graphical representation. And also, we discussed the stability analysis of the our models.
\end{abstract}

\section{Introduction}

One the most important milestones for research in modern Cosmology as well as gravitational physics is observed late time acceleration of the Universe, Which is indicated by observational data from the Cosmic microwave background [1], observations of Type Ia Supernovae [2,3] and large scale structure [4]. In Ref. [5,6] believed that dark energy (DE) occupies $73 \%$ of the Universe, dark matter (DM) occupies $23 \%$, and rest of the energy $4 \%$ is baryonic matter. In order to the details of this late time acceleration, two different approaches have been advocated: (i) to construct various DE candidate and (ii) the modification of Einstein theory of gravitation. Among the several modifications of the theories Brans-Dicke (BD) [7] and Saez-Ballester (SB)[8] scalar- tensor theories play vital role. In literatures [9-15], there are very interesting reviews on both dynamical DE models and modified theories. Several dynamical DE models include a family of scalar field such as quintessence [16-19], Phantom [20-23], Quintom [24,25], Tachon [26,27], K-Essence [28], Chaplygin gas and modified Chaplygin gas [29-44] models have been developed.

Amongst various DE candidates, the holographic DE (HDE) and new agegraphic DE, Tsallis holographic DE (THDE), which contain some significant properties of the quantum gravity have drawn considerable attention to solve the DE puzzle. HDE is depends on principle of holographic $[45,46]$ that states that the no. of degrees of freedom of a physical system scales with its bounding are rather than with its volume. HDE model was conjectured with Benkenstein entropy and Hubble horizon as IR cut-off which fails to produce suitable explanation for the history of a flat FRW Universe [47-49]. Li et al. [50] have performed a detailed investigation on the cosmological constraints on the holographic dark energy (HDE) model by using the Plank data. Tavayef et al. [51] have discussed about THDE model and found that the identification of IR-cutoff with the Hubble radius provides the late time accelerated Universe in the absence of interaction between two dark sectors of the Universe. Sharif et al. [52] have reconstruction paradigm for THDE model with dust fluid has been studied using generalized Tsallis entropy conjecture with Hubble horizon in the background of $f(G, T)$ gravity and the cosmological evolution through cosmic diagnostic parameters and phase planes discussed. According to Ref. [53], Tsallis HDE in BD cosmology have been studied by considering the Hubble horizon as the IR cutoff and studied the the stability analysis which shows that both the interacting and non-interacting models are classically unstable.

\footnotetext{
a e-mail: sobhan.maths@gmail.com (corresponding author)
} 
Abdollahi Zadeh et al. [54] has explained a short note on THDE model. Gunjan et al. [55] has discussed Statefinder diagnosis for interacting THDE models with $\omega_{t}-\omega_{t}^{\prime}$ pair. Ghaffari et al. [53] have explained Tsallis holographic dark energy in Fractal Universe. Sadri [56] has discussed observational constraints on interacting THDE model. Aditya et al. [57] have studied observational constraint on interacting THDE in logarithmic BD theory. Sharif and Saba [58] have established a reconstruction scenario for THDE model in the background of modified theory of gravity with the Hubble horizon as well as the generalised Tsallis entropy conjecture using the powerlaw solution of the scale factor.

The Bianchi type (BT) models are the nice and simplest anisotropic models, which completely explain the anisotropic effects. Mishra et al. [59] have investigated BT$\mathrm{V}$ string cosmological model with anisotropic distribution of DE. Aditya and Reddy [60] have studied BT-I string models in modified theory of gravity. Sharif et al. [61] have investigated BT-I new HDE model in the background of BD theory of gravity. Aditya and Reddy [62] have investigated anisotropic new HDE model in the framework of SB theory of gravitation.

Chandra et al. [63] have studied THDE in BT-I Universe using hybrid expansion law with k-essence. Korunur [64] has studied THDE model in Bianchi type-III Universe with scalar fields. Zadeh et al. [65] explained the cosmic evolution of THDE in BT-I model filled by DE and DM interacting with each other throughout a sign-changeable interaction with various IR cut-offs. Recently, Prasanthi and Aditya [66] have investigated BT-VI ${ }_{0}$ Renyi HDE models in the frame-work of general relativity. Very recently, Santhi and Sobhanbabu $[67,68]$ have studied BT-III and BT-VI $\mathrm{I}_{0}$ THDE models in Scalar tensor theories of gravitation.

In this work, inspired by the above investigations, we have considered the Kantowski-Sachs Universe THDE model with SB theory of gravitation. The organization of this work is as follows: In the next section, we have derived SB field equations with the help of kantowski-Sachs in the presence of two interacting fields: DM and THDE components. Also, devoted to the cosmological solution of the field equations. In Sect. 3, we study the evolution of the Universe by considering an non-interaction and sign-changeable interaction between DM and THDE whose IR cut-offs are the apparent horizon. Finally, in last section, we presented the conclusions of this work.

\section{Metric and SB field equations}

We consider a homogeneous and anisotropic KantowskiSachs (KK) Universe described by the line-element

$d s^{2}=d t^{2}-A^{2}(t) d r^{2}-B^{2}(t)\left(d \theta^{2}+\sin ^{2} \theta d \phi^{2}\right)$,

where $A(t)$ and $B(t)$ are functions of cosmic time $t$ only. For the Universe filled by a DM without pressure with energy density $\left(\rho_{M}\right)$, and DE candidate with energy density $\left(\rho_{T}\right)$, The SB field equations are

$G_{i j}-w \phi^{n}\left(\phi_{, i} \phi_{, j}-\frac{1}{2} g_{i j} \phi_{, k} \phi^{, k}\right)=-\left(T_{i j}+\bar{T}_{i j}\right)$,

where $T_{i j}$ and $\bar{T}_{i j}$ are energy momentum tensors (EMT) for $\mathrm{DM}$ and DE respectively. Scalar field $\phi$ equation

$2 \phi^{n} \phi_{, i}^{, i}+n \phi^{n-1} \phi_{, k} \phi^{, k}=0$,

and energy conservation equations are

$T_{i j}+\bar{T}_{i j}=0$.

The EMT for DM $\left(T_{i j}\right)$ and anisotropic DE are given by

$$
\begin{aligned}
& T_{i j}=\operatorname{diag}[1,0,0,0] \rho_{M}, \text { and } \\
& \bar{T}_{i j}=\operatorname{diag}\left[1,-\omega_{T},-\left(\omega_{T}+\gamma\right),-\left(\omega_{T}+\gamma\right)\right] \rho_{T},
\end{aligned}
$$

where $\omega_{T}=\frac{p_{T}}{\rho_{T}}$ is equation of state $(\mathrm{EoS})$ parameter, $p_{t}$ represents pressure of THDE and $\alpha$ is skewness parameter is in deviation from EoS parameter $\omega_{T}$ on $y$ and $z$ axes respectively.

The SB field Eq. (2), for KK line-element Eq. (1) with the help of Eq. (5), can be written as

$2 \frac{\ddot{B}}{B}+\frac{\dot{B}^{2}}{B^{2}}+\frac{1}{B^{2}}-\frac{w}{2} \phi^{n} \dot{\phi}^{2}=-\omega_{T} \rho_{T}$,

$\frac{\ddot{A}}{A}+\frac{\ddot{B}}{B}+\frac{\dot{A} \dot{B}}{A B}-\frac{w}{2} \phi^{n} \dot{\phi}^{2}=-\left(\omega_{T}+\alpha\right) \rho_{T}$,

$2 \frac{\dot{A} \dot{B}}{A B}+\frac{\dot{B}^{2}}{B^{2}}+\frac{1}{B^{2}}+\frac{w}{2} \phi^{n} \dot{\phi}^{2}=\rho_{M}+\rho_{T}$,

$\ddot{\phi}+\left(\frac{\dot{A}}{A}+2 \frac{\dot{B}}{B}\right) \dot{\phi}+\frac{n}{2} \frac{\dot{\phi}^{2}}{\phi}=0$.

We can write the continuity Eq. (4) of the DM and DE as

$$
\begin{aligned}
& \dot{\rho_{M}}+\left(\frac{\dot{A}}{A}+2 \frac{\dot{B}}{B}\right) \rho_{m}+\dot{\rho_{T}}+\left(\frac{\dot{A}}{A}+2 \frac{\dot{B}}{B}\right)\left(1+\omega_{T}\right) \rho_{T} \\
& +2 \frac{\dot{B}}{B} \alpha \rho_{T}=0,
\end{aligned}
$$


where overhead dot (.) represents ordinary differentiation with respect to cosmic time $t$.

The SB field equations Eqs. (6)-(8) form a system of four non-linear equations with seven (7) unknowns: $A, B, \rho_{M}$, $\rho_{T}, \omega_{T}, \alpha$, and $\phi$. Hence to find a deterministic solution of the non linear equations we use the condition that the shear scalar $\sigma^{2}$ is directly proportional to scalar expansion $\theta$ which leads to a relation between the metric potentials so that in Ref. [69] we have

$A=B^{k}$,

where $k \neq 1$ is positive constant. From Eqs. (6), (7), and (11), we get

$$
\frac{\ddot{B}}{B}+(1+k) \frac{\dot{B}}{B}=\frac{1}{k-1}\left(\alpha \rho_{T}+\frac{1}{B^{2}}\right) \frac{B}{\dot{B}} .
$$

To get the solution of the models we consider the ref $[70,71]$

$\alpha=\frac{\alpha_{0}(k-1) \dot{B} B-1}{B^{2} \rho_{T}}$,

where $\alpha_{0}$ is an arbitrary constant. Now from equations (12) and (13), we obtain the metric potentials as

$$
\begin{aligned}
& A=\left[\frac{\alpha_{1}(k+2)}{\alpha_{0}} e^{\alpha_{0} t}+\alpha_{2}(k+2)\right]^{\frac{k}{k+2}}, \\
& B=\left[\frac{\alpha_{1}(k+2)}{\alpha_{0}} e^{\alpha_{0} t}+\alpha_{2}(k+2)\right]^{\frac{1}{k+2}},
\end{aligned}
$$

where $\alpha_{1}$ and $\alpha_{2}$ is an constants of integrations. The lineelement Eq (1) can, be written as

$$
\begin{aligned}
d s^{2}= & d t^{2}-\left[\frac{\alpha_{1}(k+2)}{\alpha_{0}} e^{\alpha_{0} t}+\alpha_{2}(k+2)\right]^{\frac{2 k}{k+2}} d r^{2} \\
& -\left[\frac{\alpha_{1}(k+2)}{\alpha_{0}} e^{\alpha_{0} t}+\alpha_{2}(k+2)\right]^{\frac{2}{k+2}}\left(d \theta^{2}+\sin ^{2} \theta d \phi^{2}\right) .
\end{aligned}
$$

Equation (15) describes KK THDE cosmological model in SB scalar tensor theory of gravitation.

The Hubble parameter $H$ for our model can be obtain as

$H=\frac{1}{3}\left(\frac{\dot{A}}{A}+2 \frac{\dot{B}}{B}\right)=\frac{\alpha_{1}}{\left(\frac{\alpha_{1}}{\alpha_{0}}+\alpha_{2} e^{-\alpha_{0} t}\right)}$

The THDE has been proposed by Tavayef et al., [72]. The energy density of THDE is can be written as

$\rho_{T}=\gamma_{1} H^{4-2 \delta}=\left[\frac{\alpha_{1}}{\left(\frac{\alpha_{1}}{\alpha_{0}}+\alpha_{2} e^{-\alpha_{0} t}\right)}\right]^{4-2 \delta}$,

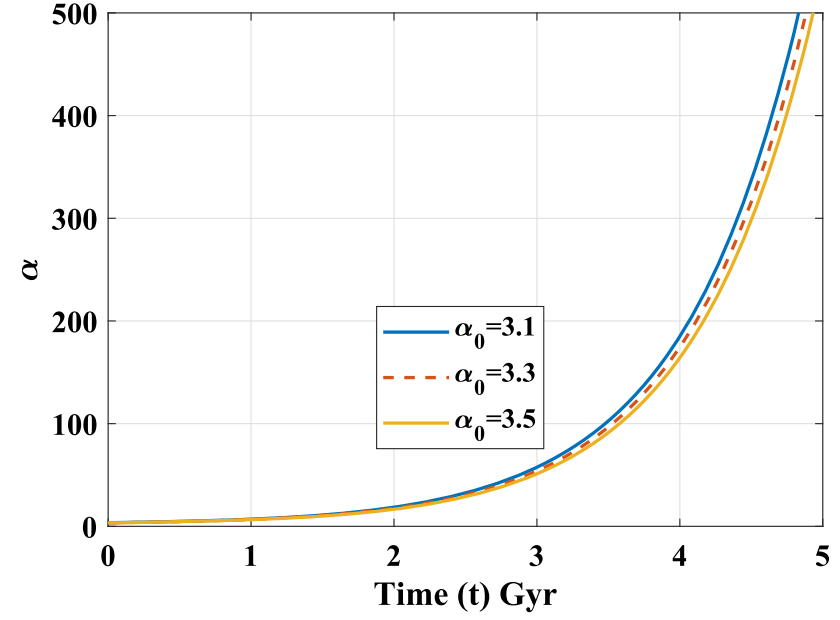

Fig. 1 Plot of skewness parameter $(\alpha)$ versus time $(t)$ for $k=1.9$, $\alpha_{1}=1.2, \alpha_{2}=0.52, w=1000, \phi_{0}=1$, and $\gamma_{1}=101$

where $\gamma_{1}$ and $\delta$ are constants.

\section{Non-interacting THDE in the SB cosmology}

In this case, we consider that there is no energy exchange between the cosmos sectors (DM, THDE), and hence, the energy conservation equations can be written in separately, so that we have from Eq. (10),

$\dot{\rho}_{M}+3 H \rho_{M}=0$,

and

$\dot{\rho}_{T}+3 H\left(1+\omega_{T}\right) \rho_{T}+2 \alpha \rho_{T} \frac{\dot{B}}{B}=0$

From Eq. (9), we get

$\phi^{\frac{n+2}{2}}=\left(\frac{n+2}{2}\right) \phi_{0}$
$\quad \times \int\left[\frac{\alpha_{1}(k+2)}{\alpha_{0}} e^{\alpha_{0} t}+\alpha_{2}(k+2)\right]^{-3} d t+\phi_{1}$,

where $\phi_{0}$ and $\phi_{1}$ are constants of integration. Taking the cosmic time derivative of Eq. (17), we have

$\dot{\rho}_{T}=\gamma_{1}(4-2 \delta) H^{3-2 \delta} \dot{H}$. 


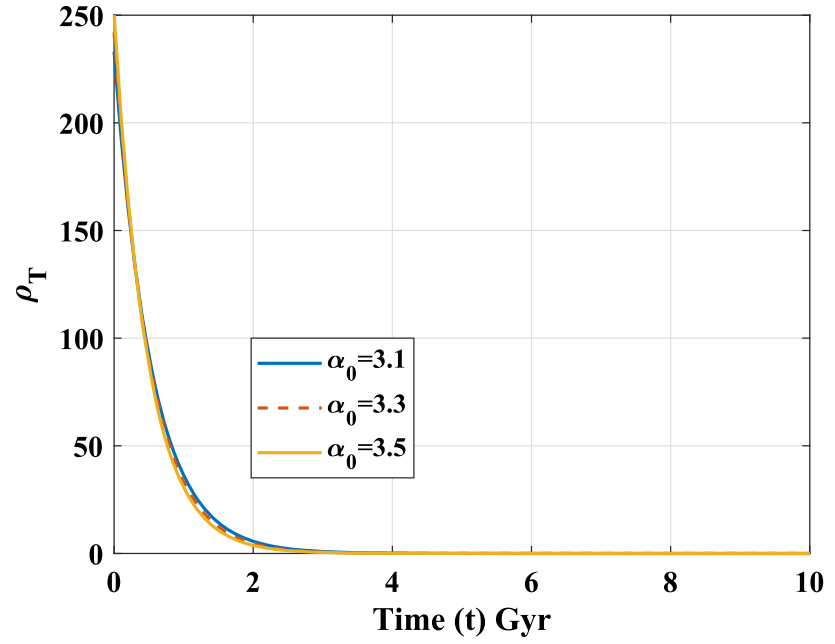

Fig. 2 Plot of energy density $\left(\rho_{T}\right)$ of THDE versus time $(t)$ for $k=1.9$, $\alpha_{1}=1.2, \alpha_{2}=0.52$, and $\gamma_{1}=101$

Now using Eqs. (14), (17), and (20) in Eq. (8), we get

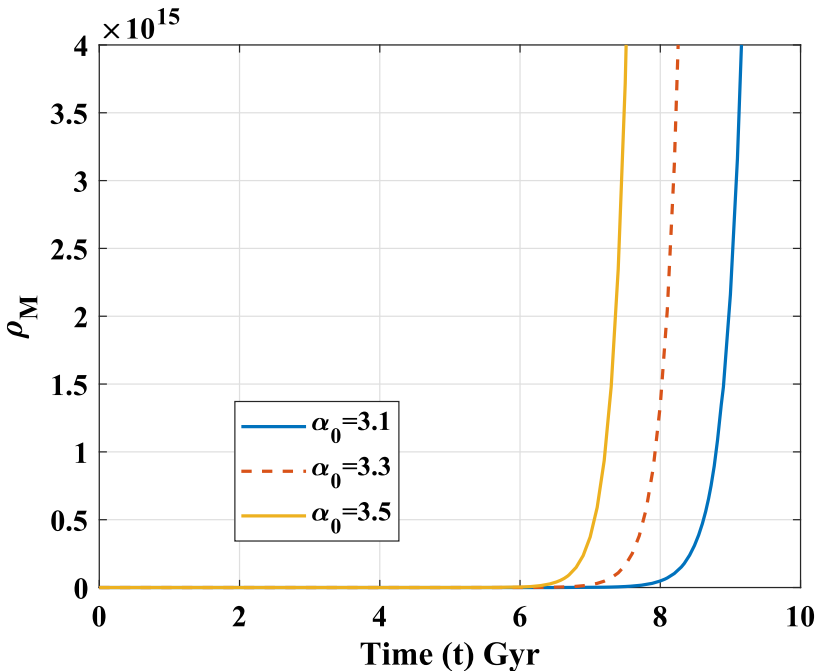

Fig. 3 Plot of energy density $\left(\rho_{M}\right)$ of DM versus time $(t)$ for $k=1.9$, $\alpha_{1}=1.2, \alpha_{2}=0.52$, and $\gamma_{1}=101$

$$
\left.\begin{array}{rl}
\rho_{M}= & \frac{(2 k+1) \alpha_{1}^{2} e^{2 \alpha_{0} t}}{\left[\frac{\alpha_{1}(k+2)}{\alpha_{0}} e^{\alpha_{0} t}+\alpha_{2}(k+2)\right]^{2}}+\frac{1}{\left[\frac{\alpha_{1}(k+2)}{\alpha_{0}} e^{\alpha_{0} t}+\alpha_{2}(k+2)\right]^{\frac{2}{2+k}}} \\
& +\frac{w}{2} \phi_{0}^{2}\left[\frac{\alpha_{1}(k+2)}{\alpha_{0}} e^{\alpha_{0} t}+\alpha_{2}(k+2)\right]^{-6}-\gamma_{1}\left[\frac{\alpha_{1}}{\frac{\alpha_{1}}{\alpha_{0}}+\alpha_{2} e^{-\alpha_{0} t}}\right]^{4-2 \delta}
\end{array}\right\}
$$

Using Eqs. (14), (17) in Eq. (13), we get skewness parameter $\alpha$ is

$\alpha=\frac{\alpha_{0} \alpha_{1}(k-1) e^{\alpha_{0} t}\left[\frac{\alpha_{1}(k+2)}{\alpha_{0}} e^{\alpha_{0} t}+\alpha_{2}(k+2)\right]^{\frac{-k}{2+k}}-1}{\gamma_{1}\left[\frac{\alpha_{1}(k+2)}{\alpha_{0}} e^{\alpha_{0} t}+\alpha_{2}(k+2)\right]^{2}\left[\frac{\alpha_{1}}{\frac{\alpha_{1}}{\alpha_{0}}+\alpha_{2} e^{-\alpha_{0} t}}\right]^{4-2 \delta}}$

From Eqs. (14), (16), (17) and (19), we get the EoS parameter $\omega_{T}$ of THDE is

$$
\left.\begin{array}{rl}
\omega_{T}= & -1+2 \alpha_{0} \alpha_{1} \alpha_{2} \gamma_{1}(\delta-2) e^{\alpha_{0} t} H^{3-2 \delta}+\frac{2 \alpha_{1}^{4 \delta-9}}{3 \gamma_{1}} \\
& \left.\times\left[1-\alpha_{0} \alpha_{1}(k-1) e^{\alpha_{0} t}\left[\frac{\alpha_{1}(k+2)}{\alpha_{0}} e^{\alpha_{0} t}+\alpha_{2}(k+2)\right]^{\frac{-k}{2+k}}\right]\right\} \\
& \times\left[\frac{\alpha_{1}(k+2)}{\alpha_{0}} e^{\alpha_{0} t}+\alpha_{2}(k+2)\right]^{-\left(\frac{4+k}{2+k}\right)} H^{5-2 \delta}
\end{array}\right\}
$$

Taking the derivative of Eq. (24) with respect to $x=\ln a$, we get

$$
\begin{aligned}
& \omega_{t}^{\prime}=2 \alpha_{0} \alpha_{1} \alpha_{2} \gamma_{1}(\delta-2) e^{\alpha_{0} t} H^{1-2 \delta}\left((3-2 \delta) \dot{H}+\alpha_{0} H\right) H^{2-2 \delta} \\
& +\frac{2 \alpha_{1}^{4 \delta-9}}{3 \gamma_{1}}\left[\frac{\alpha_{1}(k+2)}{\alpha_{0}} e^{\alpha_{0} t}+\alpha_{2}(k+2)\right]^{-\left(\frac{4+k}{2+k}\right)} H^{4-2 \delta} \\
& \times\left[\left(1-\alpha_{0} \alpha_{1}(k-1) e^{\alpha_{0} t}\left[\frac{\alpha_{1}(k+2)}{\alpha_{0}} e^{\alpha_{0} t}+\alpha_{2}(k+2)\right]^{-\left(\frac{k}{2+k}\right)}\right)\right. \\
& \left.\times\left((5-2 \delta) \frac{\dot{H}}{H}-\left(\frac{4+k}{2+k}\right)\left[\frac{\alpha_{1}(k+2)}{\alpha_{0}} e^{\alpha_{0} t}+\alpha_{2}(k+2)\right]^{-1}\right)\right] \\
& +\alpha_{0}^{2} \alpha_{1}(1-k) e^{\alpha_{0} t}\left[\left[\frac{\alpha_{1}(k+2)}{\alpha_{0}} e^{\alpha_{0} t}+\alpha_{2}(k+2)\right]^{-\frac{k}{2+k}}\right. \\
& \left.-\frac{\alpha_{1} k}{\alpha_{0}} e^{\alpha_{0} t}\left[\frac{\alpha_{1}(k+2)}{\alpha_{0}} e^{\alpha_{0} t}+\alpha_{2}(k+2)\right]^{-\left(\frac{k+1}{2+k}\right)}\right],
\end{aligned}
$$

where $\dot{H}=\frac{\alpha_{0} \alpha_{1} \alpha_{2} e^{-\alpha_{0} t}}{\left(\frac{\alpha_{0}}{\alpha_{0}}+\alpha_{2} e^{-\alpha_{0} t}\right)^{2}}$

The squared of sound speed $v_{s}^{2}$ is useful to study the stability of the model. If $v_{s}^{2}$ is positive, we obtain stable model and $v_{s}^{2}$ is negative, we obtain unstable model. In this case $v_{s}^{2}$ takes the form 


$$
\begin{aligned}
v_{s}^{2}= & -1+2 \alpha_{0} \alpha_{1} \alpha_{2} \gamma_{1}(\delta-2) e^{\alpha_{0} t} H^{3-2 \delta}+\frac{2 \alpha_{1}^{4 \delta-9}}{3 \gamma_{1}}\left[1-\alpha_{0} \alpha_{1}(k-1) e^{\alpha_{0} t}\left[\frac{\alpha_{1}(k+2)}{\alpha_{0}} e^{\alpha_{0} t}\right.\right. \\
& \left.\left.+\alpha_{2}(k+2)\right]^{\frac{-k}{2+k}}\right]\left[\frac{\alpha_{1}(k+2)}{\alpha_{0}} e^{\alpha_{0} t}+\alpha_{2}(k+2)\right]^{-\left(\frac{4+k}{2+k}\right)} H^{5-2 \delta}+2 \alpha_{0} \alpha_{1} \alpha_{2} \gamma_{1}(\delta-2) e^{\alpha_{0} t} H^{1-2 \delta} \\
& \times\left((3-2 \delta) \dot{H}+\alpha_{0} H\right) \frac{H^{3-2 \delta}}{(4-2 \delta) \dot{H}}+\frac{2 \alpha_{1}^{4 \delta-9} H^{6-2 \delta}}{3 \gamma_{1}(4-2 \delta) \dot{H}}\left[\frac{\alpha_{1}(k+2)}{\alpha_{0}} e^{\alpha_{0} t}+\alpha_{2}(k+2)\right]^{-\left(\frac{4+k}{2+k}\right)} \\
& \times\left[( 1 - \alpha _ { 0 } \alpha _ { 1 } ( k - 1 ) e ^ { \alpha _ { 0 } t } [ \frac { \alpha _ { 1 } ( k + 2 ) } { \alpha _ { 0 } } e ^ { \alpha _ { 0 } t } + \alpha _ { 2 } ( k + 2 ) ] ^ { - ( \frac { k } { 2 + k } ) } ) \left((5-2 \delta) \frac{\dot{H}}{H}\right.\right. \\
& \left.-\left(\frac{4+k}{2+k}\right)\left[\frac{\alpha_{1}(k+2)}{\alpha_{0}} e^{\alpha_{0} t}+\alpha_{2}(k+2)\right]^{-1}\right)+\alpha_{0}^{2} \alpha_{1}(1-k) e^{\alpha_{0} t}\left[\left[\frac{\alpha_{1}(k+2)}{\alpha_{0}} e^{\alpha_{0} t}+\alpha_{2}(k+2)\right]^{-\frac{k}{2+k}}\right. \\
& \left.\left.-\frac{\alpha_{1} k}{\alpha_{0}} e^{\alpha_{0} t}\left[\frac{\alpha_{1}(k+2)}{\alpha_{0}} e^{\alpha_{0} t}+\alpha_{2}(k+2)\right]^{-\left(\frac{k+1}{2+k}\right)}\right]\right] .
\end{aligned}
$$

In order to understand the role of skewness parameter $\alpha$ and in the evolution of cosmos, we analyze the dynamical parameters through graphical representation for various values of $\alpha_{0}=3.1,3.3,3.5$. The graphical nature of skewness parameter $\alpha$ versus cosmic time $t$ for THDE model with Hubble horizon cut-off is shown in Fig. 1. It is observed that skewness parameter $\alpha$ varies in the positive region and do not vanish throughout its evolution for chosen values of $\alpha_{0}$.

Figure 2 represents the plot of energy density of THDE with Hubble horizon cut-off against cosmic time $t$ for different values of $\alpha_{0}$. The trajectories of $\rho_{T}$ indicates that the positive behavior for the values of $\alpha_{0}=3.1,3.3,3.5$. It can be seen that $\rho_{T}$ decreases with increases of $\alpha_{0}$. And also, we observed that in the beginning suggest that the energy density $\rho_{T}$ dominates the early Universe but for large cosmic time $t$ $\rho_{T}$ vanish (negligible).

According to Fig. 5, we have drawn $\omega_{T}-\omega_{T}^{\prime}$ plane. we can see $\omega_{T}<0$ and $\omega_{T}^{\prime}>0$ for different values $\alpha_{0}=$ $3.1,3.3,3.5$. Hence, our model completely lies in the thawing region.

The plot of energy density of DM versus cosmic time $t$ for different values of $\alpha_{0}$ is shown in Fig. 3. It can be seen that $\rho_{M}$ is positive and increasing function of time $t$ for the values of $\alpha_{0}=3.1,3.3,3.5$. We can also, observed that the energy density of DM $\rho_{M}$ increases with decreases of $\alpha_{0}$. This behavior is opposite to the behavior energy density of THDE $\rho_{T}$.

The behavior of EoS parameter versus cosmic time $t$ for the non-interacting THDE model is depicted in Fig. 4 for different values of $\alpha_{0}$. It may be observe that the model starts in matter dominated region and varies in quintessence region and finally, its reached to LCDM model for the values of $\alpha_{0}=3.1,3.3,3.5$.
According to Figs. 10, 11 and 12, we can observe that $\omega_{T}$ is negative and $\omega_{T}^{\prime}$ is positive for different values of $\alpha_{0}$ and $\beta$. Hence, our model completely in the thawing region.

According to Fig. 6, we shown that $v_{s}^{2}$ versus cosmic time $t$ we can observed that $v_{s}^{2}$ is negative, which shows that our model is unstable.

\subsection{Sign-changeable interaction}

In the KK anisotropic background, filled with DM and THDE interacting with each other, The EM conservation law Eq. (10) is separated into

$\dot{\rho_{M}}+3 H \rho_{M}=Q$

and

$\dot{\rho_{T}}+3 H\left(1+\omega_{T}\right) \rho_{T}+2 \alpha \rho_{T} \frac{\dot{B}}{B}=-Q$,

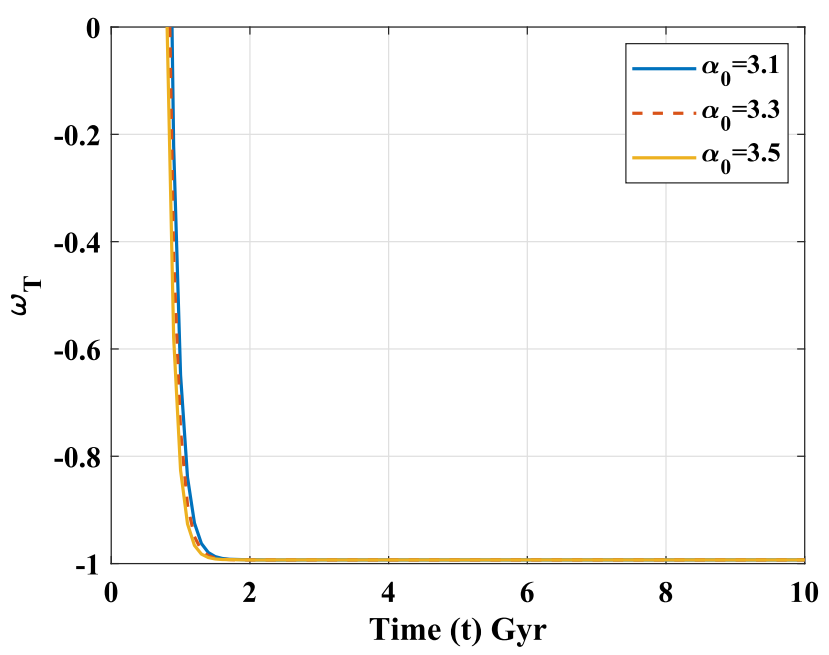

Fig. 4 Plot of EoS parameter $\left(\omega_{T}\right)$ versus time $(t)$ for $k=1.9, \alpha_{1}=$ $1.2, \alpha_{2}=0.52$, and $\gamma_{1}=101$ 


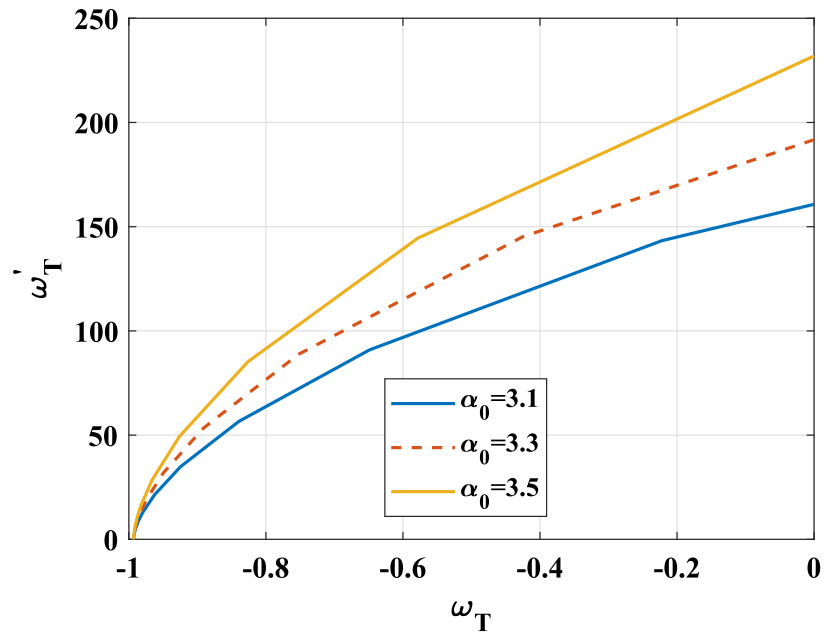

Fig. 5 Plot of $\omega_{T}$ versus $\omega_{T}^{\prime}$ for $k=1.9, \alpha_{1}=1.2, \alpha_{2}=0.52$, and $\gamma_{1}=101$

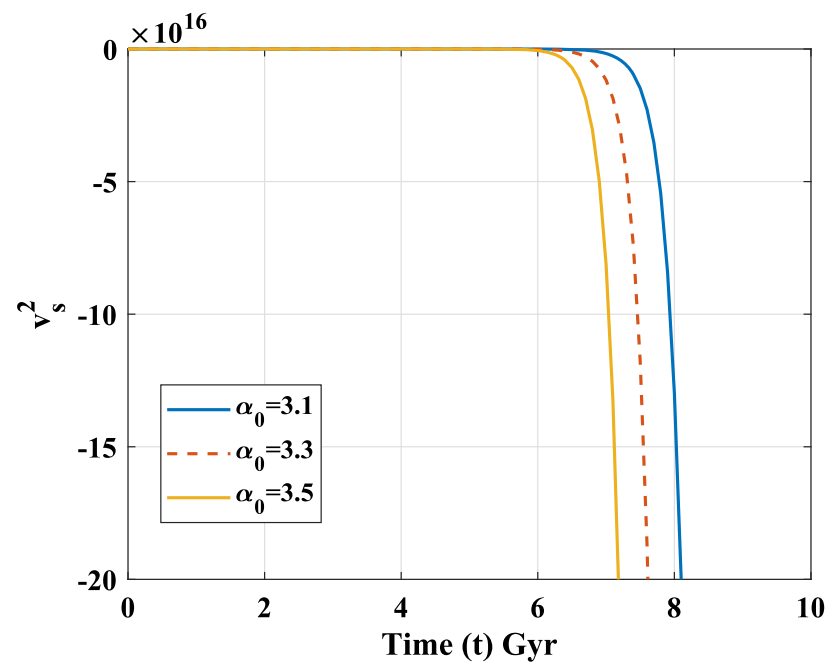

Fig. 6 Plot of $v_{s}^{2}$ versus time $(t)$ for $k=1.9, \alpha_{1}=1.2, \alpha_{2}=0.52$, and $\gamma_{1}=101$

where $Q$ represents the interaction term, and assume that it has the $Q=3 \beta H q \rho_{T}$ from the ref. [73-75]. Here $\beta$ is a coupling constant and this $\beta$ should assumed to be negative because if $\beta$ is positive then the result in $\rho_{T}$ is negative. Definitely, as the expansion of the Universe changes from deceleration $q>0$ to acceleration $q<0$. $Q$ can be changing from negative to positive.

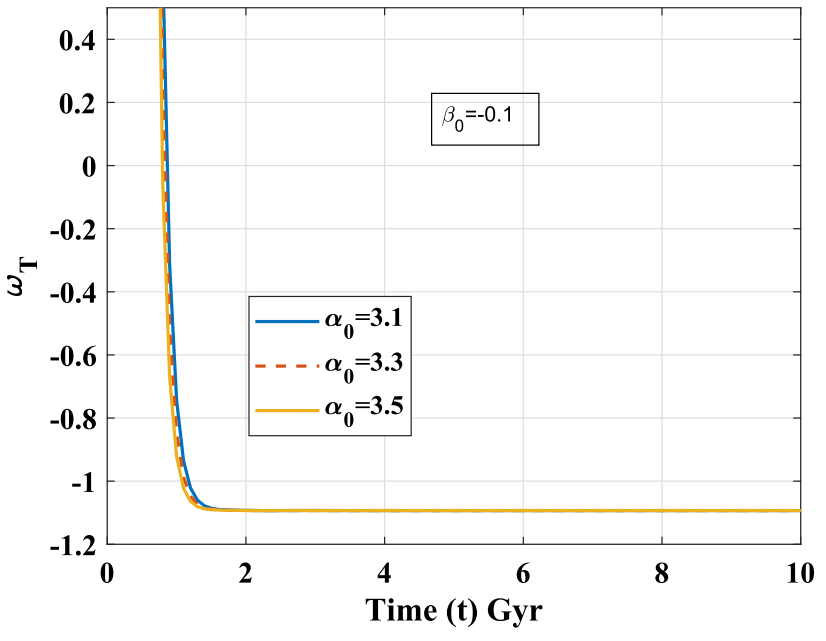

Fig. 7 Plot of EoS parameter $\left(\omega_{T}\right)$ versus time $(t)$ for $k=1.9, \alpha_{1}=$ $1.2, \alpha_{2}=0.52, \gamma_{1}=101$, and $\beta=-0.1$

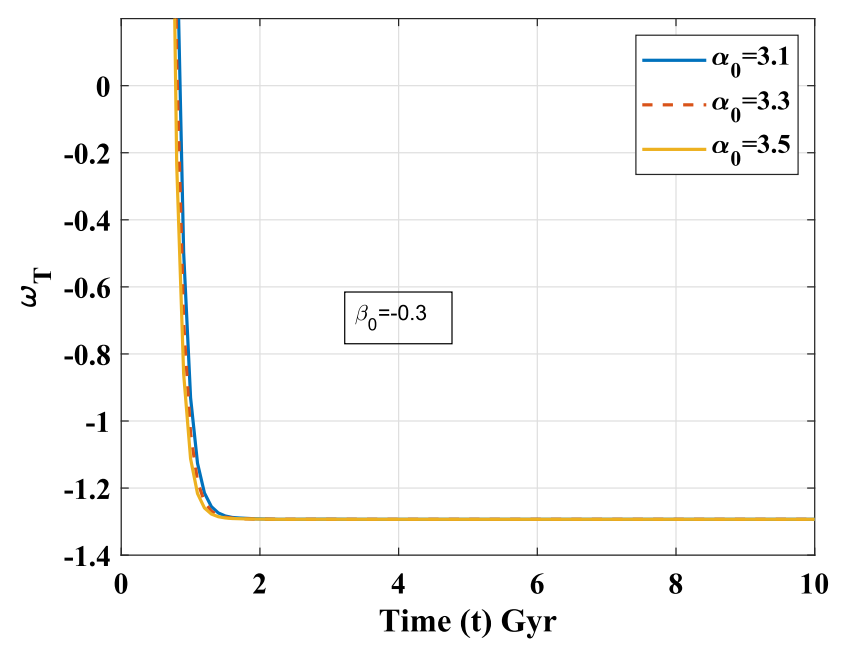

Fig. 8 Plot of EoS parameter $\left(\omega_{T}\right)$ versus time $(t)$ for $k=1.9, \alpha_{1}=$ $1.2, \alpha_{2}=0.52, \gamma_{1}=101$, and $\beta=-0.3$

$$
\begin{aligned}
\omega_{T}= & -1+2 \alpha_{0} \alpha_{1} \alpha_{2} \gamma_{1}(\delta-2) e^{\alpha_{0} t} H^{3-2 \delta}+\frac{2 \alpha_{1}^{4 \delta-9}}{3 \gamma_{1}} \\
& \times\left[1-\alpha_{0} \alpha_{1}(k-1) e^{\alpha_{0} t}\left[\frac{\alpha_{1}(k+2)}{\alpha_{0}} e^{\alpha_{0} t}+\alpha_{2}(k+2)\right]^{\frac{-k}{2+k}}\right] \\
& \times\left[\frac{\alpha_{1}(k+2)}{\alpha_{0}} e^{\alpha_{0} t}+\alpha_{2}(k+2)\right]^{-\left(\frac{4+k}{2+k}\right)} H^{5-2 \delta}-\beta q
\end{aligned}
$$

Taking the derivative of Eq. (29) with respect to $x=\ln a$, we get 


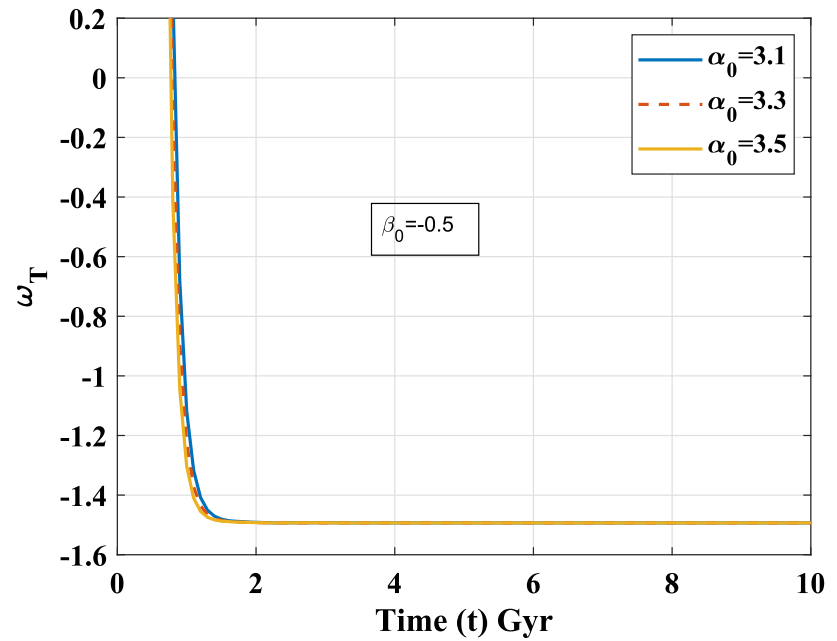

Fig. 9 Plot of EoS parameter $\left(\omega_{T}\right)$ versus time $(t)$ for $k=1.9, \alpha_{1}=$ $1.2, \alpha_{2}=0.52, \gamma_{1}=101$, and $\beta=-0.5$

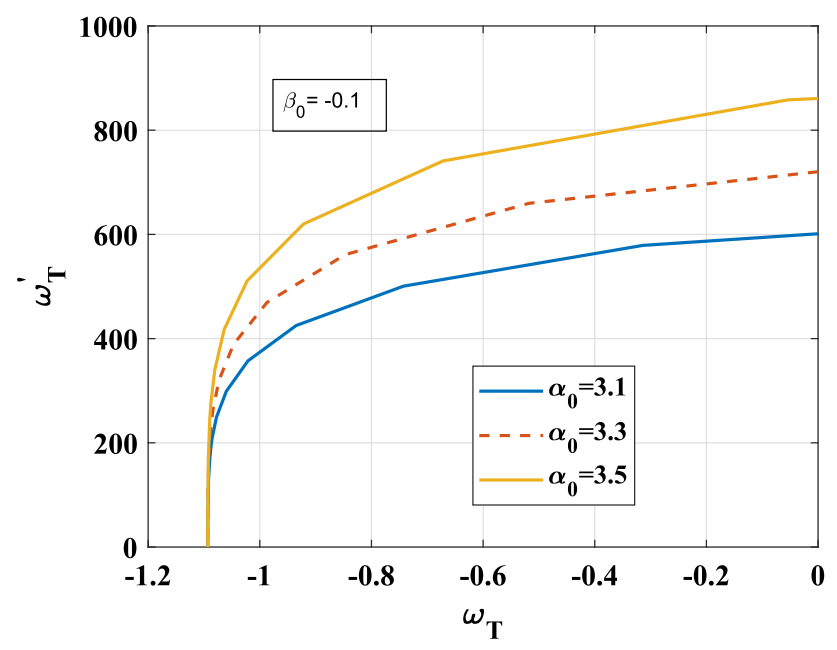

Fig. 10 Plot of $\omega_{T}$ versus $\omega_{T}^{\prime}$ for $k=1.9, \alpha_{1}=1.2, \alpha_{2}=0.52$, $\gamma_{1}=101$, and $\beta=-0.1$

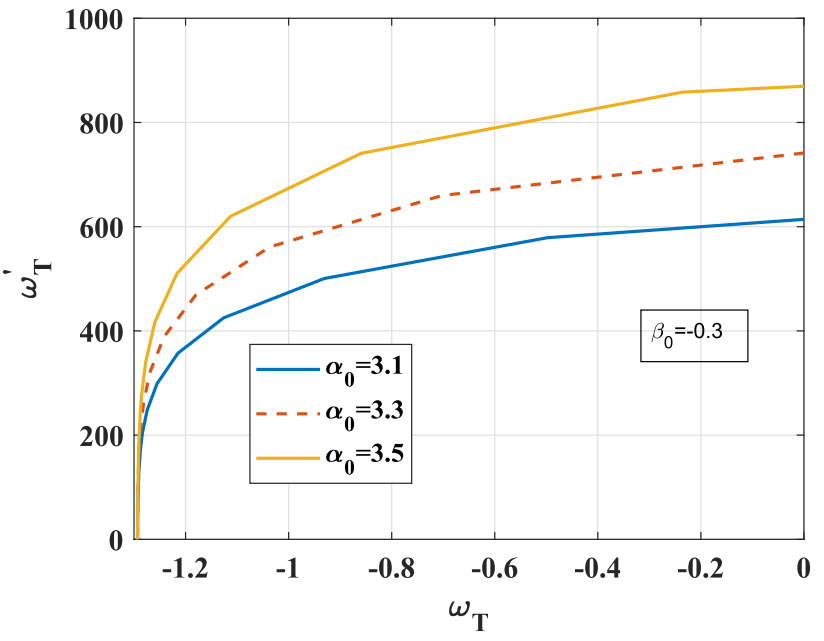

Fig. 11 Plot of $\omega_{T}$ versus $\omega_{T}^{\prime}$ for $k=1.9, \alpha_{1}=1.2, \alpha_{2}=0.52$, $\gamma_{1}=101$, and $\beta=-0.3$

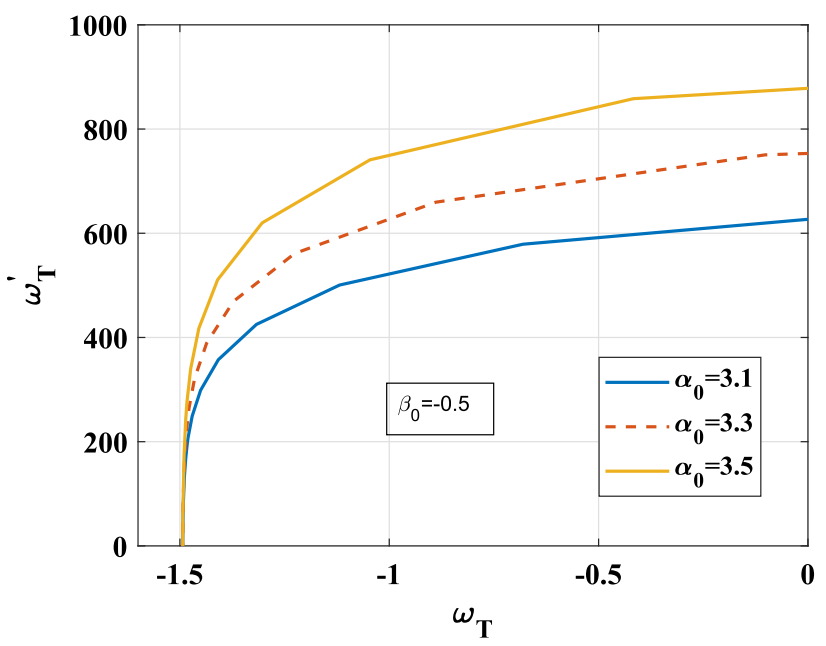

Fig. 12 Plot of $\omega_{T}$ versus $\omega_{T}^{\prime}$ for $k=1.9, \alpha_{1}=1.2, \alpha_{2}=0.52$, $\gamma_{1}=101$, and $\beta=-0.5$

where $\dot{q}=-\frac{\alpha_{0}^{2} \alpha_{2} e^{-\alpha_{0} t}}{\alpha_{1}}$.

$$
\begin{aligned}
\omega_{t}^{\prime}= & 2 \alpha_{0} \alpha_{1} \alpha_{2} \gamma_{1}(\delta-2) e^{\alpha_{0} t} H^{1-2 \delta}\left((3-2 \delta) \dot{H}+\alpha_{0} H\right) H^{2-2 \delta} \\
& +\frac{2 \alpha_{1}^{4 \delta-9}}{3 \gamma_{1}}\left[\frac{\alpha_{1}(k+2)}{\alpha_{0}} e^{\alpha_{0} t}+\alpha_{2}(k+2)\right]^{-\left(\frac{4+k}{2+k}\right)} H^{4-2 \delta} \\
& \times\left[\left(1-\alpha_{0} \alpha_{1}(k-1) e^{\alpha_{0} t}\left[\frac{\alpha_{1}(k+2)}{\alpha_{0}} e^{\alpha_{0} t}+\alpha_{2}(k+2)\right]^{-\left(\frac{k}{2+k}\right)}\right)\right. \\
& \left.\times\left((5-2 \delta) \frac{\dot{H}}{H}-\left(\frac{4+k}{2+k}\right)\left[\frac{\alpha_{1}(k+2)}{\alpha_{0}} e^{\alpha_{0} t}+\alpha_{2}(k+2)\right]^{-1}\right)\right] \\
& +\alpha_{0}^{2} \alpha_{1}(1-k) e^{\alpha_{0} t}\left[\left[\frac{\alpha_{1}(k+2)}{\alpha_{0}} e^{\alpha_{0} t}+\alpha_{2}(k+2)\right]^{-\frac{k}{2+k}}\right. \\
& \left.-\frac{\alpha_{1} k}{\alpha_{0}} e^{\alpha_{0} t}\left[\frac{\alpha_{1}(k+2)}{\alpha_{0}} e^{\alpha_{0} t}+\alpha_{2}(k+2)\right]^{-\left(\frac{k+1}{2+k}\right)}\right]-\beta \dot{q},
\end{aligned}
$$


The squared sound speed $v_{s}^{2}$ is obtained as

$$
\begin{aligned}
v_{s}^{2}= & -1+2 \alpha_{0} \alpha_{1} \alpha_{2} \gamma_{1}(\delta-2) e^{\alpha_{0} t} H^{3-2 \delta}+\frac{2 \alpha_{1}^{4 \delta-9}}{3 \gamma_{1}}\left[1-\alpha_{0} \alpha_{1}(k-1) e^{\alpha_{0} t}\left[\frac{\alpha_{1}(k+2)}{\alpha_{0}} e^{\alpha_{0} t}\right.\right. \\
& \left.\left.+\alpha_{2}(k+2)\right]^{\frac{-k}{2+k}}\right]\left[\frac{\alpha_{1}(k+2)}{\alpha_{0}} e^{\alpha_{0} t}+\alpha_{2}(k+2)\right]^{-\left(\frac{4+k}{2+k}\right)} H^{5-2 \delta}+2 \alpha_{0} \alpha_{1} \alpha_{2} \gamma_{1}(\delta-2) e^{\alpha_{0} t} H^{1-2 \delta} \\
& \times\left((3-2 \delta) \dot{H}+\alpha_{0} H\right) \frac{H^{3-2 \delta}}{(4-2 \delta) \dot{H}}+\frac{2 \alpha_{1}^{4 \delta-9} H^{6-2 \delta}}{3 \gamma_{1}(4-2 \delta) \dot{H}}\left[\frac{\alpha_{1}(k+2)}{\alpha_{0}} e^{\alpha_{0} t}+\alpha_{2}(k+2)\right]^{-\left(\frac{4+k}{2+k}\right)} \\
\times & {\left[( 1 - \alpha _ { 0 } \alpha _ { 1 } ( k - 1 ) e ^ { \alpha _ { 0 } t } [ \frac { \alpha _ { 1 } ( k + 2 ) } { \alpha _ { 0 } } e ^ { \alpha _ { 0 } t } + \alpha _ { 2 } ( k + 2 ) ] ^ { - ( \frac { k } { 2 + k } ) } ) \left((5-2 \delta) \frac{\dot{H}}{H}\right.\right.} \\
& \left.-\left(\frac{4+k}{2+k}\right)\left[\frac{\alpha_{1}(k+2)}{\alpha_{0}} e^{\alpha_{0} t}+\alpha_{2}(k+2)\right]^{-1}\right)+\alpha_{0}^{2} \alpha_{1}(1-k) e^{\alpha_{0} t}\left[\left[\frac{\alpha_{1}(k+2)}{\alpha_{0}} e^{\alpha_{0} t}\right.\right. \\
& \left.\left.\left.+\alpha_{2}(k+2)\right]^{-\frac{k}{2+k}}-\frac{\alpha_{1} k}{\alpha_{0}} e^{\alpha_{0} t}\left[\frac{\alpha_{1}(k+2)}{\alpha_{0}} e^{\alpha_{0} t}+\alpha_{2}(k+2)\right]^{-\left(\frac{k+1}{2+k}\right)}\right]\right]-\frac{\beta \dot{q}}{H} .
\end{aligned}
$$

The behavior of EoS parameter $\omega_{T}$ versus cosmic time $t$ for interacting THDE model is depicted in Figs. 7, 8 and 9 for the different values of $\alpha_{0}$ and $\beta$. It can be that our model starts in radiation region and passes through matter dominated region and enters into the quintessence phase while the model crosses the phantom divide line and finally, its reached to the constant value in phantom region.

According to Fig. 13, we can say that $v_{s}^{2}$ is negative, which shows that our model unstable. The rate of acceleration or deceleration of the Universe can be described by a single parameter $(q)$ is $q=-1-\frac{\dot{H}}{H^{2}}$ a more sensitive discriminator of the expansion rate and hence THDE can be constructed by considering the general form for the expansion factor of the Universe. for our model DP is

$q=-1+\frac{\alpha_{0} \alpha_{2}}{\alpha_{1}} e^{-\alpha_{0} t}$

According to Fig. 14, we can observed that our model exhibits a smooth transition from decelerating to accelerating region of the Universe.

The statefinder indicative $(r, s)$ pair is taken for the geometric idea of the models. We can find the difference in different THDE models by state-finder analysis.
According to Fig. 15, we can observe that the trajectories of $(r, s)$ plane gives a correspondence with Chaplygin gas model for $r>1$ and $s<0$.

\section{Conclusions}

We have investigated the THDE in the spatially homogeneous anisotropic KK Universe within the frame-work of SB scalar-tensor theory of gravity filled with DM and THDE. To obtainthe deterministic solution of the model of the Universe, we consider some physically plausible conditions, these conditions lead to a varying deceleration parameter which represents decelerating to accelerating expansion of the Universe. We have summarized the conclusions as follows:

$$
\begin{aligned}
& r=-\frac{\alpha_{0}^{2} \alpha_{2} e^{-\alpha_{0} t}}{\alpha_{1}^{2}}\left[\frac{\alpha_{1}}{\alpha_{0}}+\alpha_{2}\left(1+2 e^{-\alpha_{0} t}\right) \alpha_{2}\right]+\frac{3 \alpha_{0} \alpha_{2} e^{-\alpha_{0} t}}{\alpha_{1}}-6, \\
& s=2\left[\frac{-\alpha_{0}^{2} \alpha_{2} e^{-\alpha_{0} t}\left[\frac{\alpha_{1}}{\alpha_{0}}+\alpha_{2}\left(1+2 e^{-\alpha_{0} t}\right) \alpha_{2}\right]+3 \alpha_{0} \alpha_{1} \alpha_{2} e^{-\alpha_{0} t} \alpha_{1}-6 \alpha_{1}^{2}}{3 \alpha_{1}\left(2 \alpha_{0} \alpha_{2} e^{-\alpha_{0} t}-3 \alpha_{1}\right)}\right]
\end{aligned}
$$




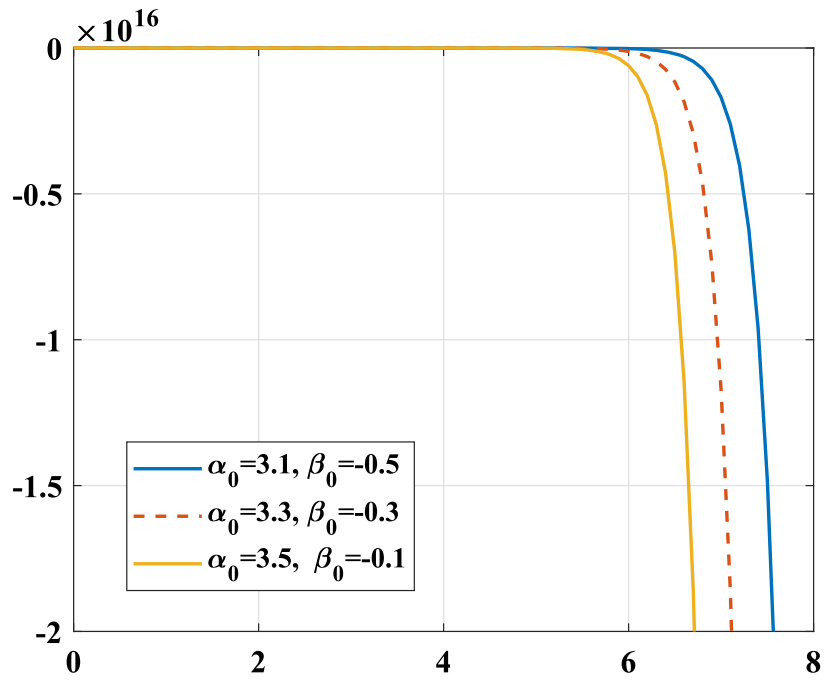

Fig. 13 Plot of $v_{s}^{2}$ versus time $(t)$ for $k=1.9, \alpha_{1}=1.2, \alpha_{2}=0.52$, $\gamma_{1}=101$, and $\beta=-0.1,-0.3,-0.5$

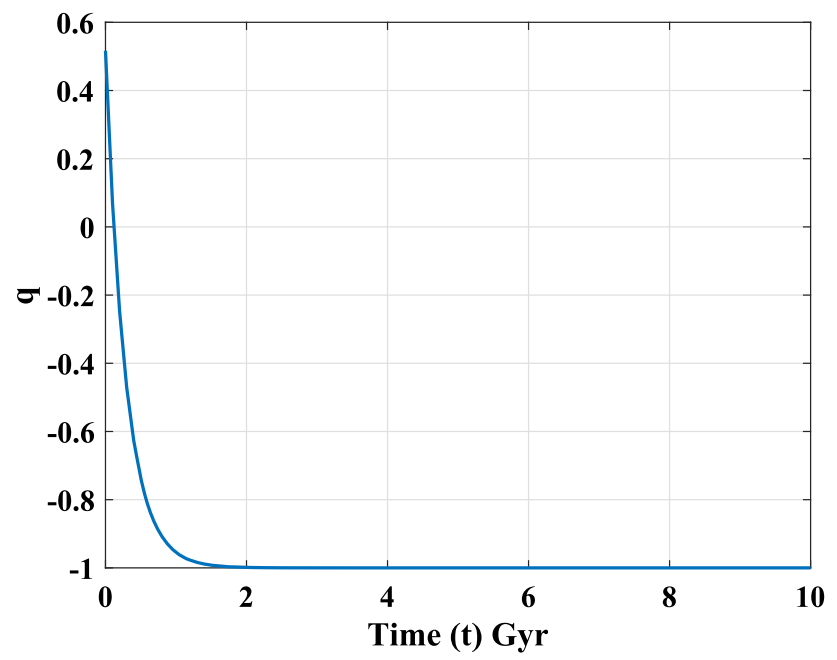

Fig. 14 Plot of $v_{s}^{2}$ versus time $(t)$ for $k=1.9, \alpha_{1}=1.2, \alpha_{2}=0.52$, and $\gamma_{1}=101$

- For our models, we have observed that skewness parameter $\alpha$ varies in the positive region and do not vanish throughout its evolution for chosen values of $\alpha_{0}=$ 3.1, 3.3, 3.5. From the trajectories of $\rho_{T}$ indicates that the positive behavior for the values of $\alpha_{0}=3.1,3.3,3.5$. And energy density of THDE $\left(\rho_{T}\right)$ decreases with increases of $\alpha_{0}$. And also, we observed that in the begining suggest that the energy density $\rho_{T}$ dominates the early Universe but for large cosmic time $(t) \rho_{T}$ is negligible.

- The energy density of DM $\rho_{M}$ is positive and increasing function of time $t$ for the various values of $\alpha_{0}$. We can also, observed that the energy density of DM $\rho_{M}$ increases with decreases of $\alpha_{0}$. This behavior is opposite to the behavior energy density of THDE $\rho_{T}$.

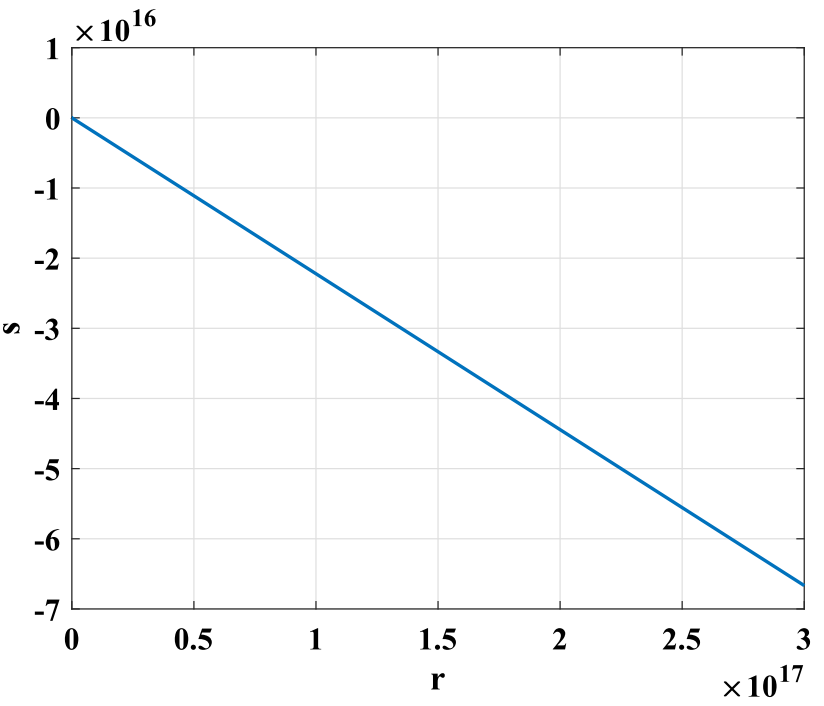

Fig. 15 Plot of $v_{s}^{2}$ versus time $(t)$ for $k=1.9, \alpha_{1}=1.2, \alpha_{2}=0.52$, and $\gamma_{1}=101$

- For our non-interacting THDE model, the behavior of EoS parameter for different values of $\alpha_{0}$. It may be observe that the model starts in matter dominated region and varies in quintessence region and finally, its reached to LCDM model.

- We have drawn $\omega_{T}-\omega_{T}^{\prime}$ plane (From the Fig. 5). We can observed $\omega_{T}<0$ and $\omega_{T}^{\prime}>0$ for different values $\alpha_{0}$. Our model completely lies in the thawing region. And the squared sound speed $v_{s}^{2}$ versus cosmic time $t$, we can observed that $v_{s}^{2}$ is negative, which shows that our model is unstable.

- For our sign-changeable THDE model, the behavior of EoS parameter $\omega_{T}$ for the different values of $\alpha_{0}$ and $\beta$. It can be that our model starts in radiation region and passes through matter dominated region and enters into the quintessence phase while the model crosses the phantom divide line and finally, its reached to the constant value in phantom region.

- According to Figs. 10, 11 and 12, we can observe that $\omega_{T}$ is negative and $\omega_{T}^{\prime}$ is positive for different values of $\alpha_{0}$ and $\beta$. Hence, our model completely in the thawing region. In both cases, our models completely in the thawing region. And Fig. 13, we can say that $v_{s}^{2}$ is negative, which shows that our model unstable and also, we observed that in both cases, our models unstable.

- For both the models, DP parameter exhibits a smooth transition from decelerating to accelerating region of the Universe. The trajectories of $(r, s)$ plane gives a correspondence with Chaplygin gas model for $r>1$ and $s<0$. 
We expect the above analysis will definitely help to have a better understanding of THDE in SB scalar-tensor theory.

Data Availability Statement This manuscript has no associated data or the data will not be deposited. [Authors' comment: All data analysed during this study are presented in this published article.]

Open Access This article is licensed under a Creative Commons Attribution 4.0 International License, which permits use, sharing, adaptation, distribution and reproduction in any medium or format, as long as you give appropriate credit to the original author(s) and the source, provide a link to the Creative Commons licence, and indicate if changes were made. The images or other third party material in this article are included in the article's Creative Commons licence, unless indicated otherwise in a credit line to the material. If material is not included in the article's Creative Commons licence and your intended use is not permitted by statutory regulation or exceeds the permitted use, you will need to obtain permission directly from the copyright holder. To view a copy of this licence, visit http://creativecomm ons.org/licenses/by/4.0/.

Funded by SCOAP ${ }^{3}$.

\section{References}

1. D.N. Spergel et al., Astrophys. J. Suppl. 148, 175 (2003)

2. A.G. Riess et al., Astron. J. 116, 1009 (1998)

3. S. Perlmutter et al., Nature 391, 51 (1998)

4. M. Tegmark et al., Phys. Rev. D 69, 103501 (2004)

5. S.M. Carroll et al., Living. Rev. Relativ. 4, 1 (2001)

6. T. Padmanabham, Phys. Rep. 380, 235 (2003)

7. C. Brans, R.H. Dicke, Phys. Rev. 124, 925 (1961)

8. D. Saez, V.J. Ballester, Phys. Lett. A 113, 467 (1986)

9. R.R. Caldwell, M. Kamionkowski, Annu. Rev. Nucl. Part. Sci. 59, 397 (2009)

10. S. Tsujikawa, Lect. Notes Phys. 800, 99 (2010)

11. S. Nojiri, S.D. Odintsov, Phys. Rep. 505, 59 (2011)

12. K. Bamba et al., Astrophys. Space Sci. 342, 155 (2012)

13. T. Clifton et al., Phys. Rep. 513, 1 (2012)

14. S. Capozziello et al., Eur. Phys. J. C 72, 2068 (2012)

15. S. Nojiri et al., Phys. Rep. 692, 1 (2017)

16. B. Ratra, P.J.E. Peebles, Phys. Rev. D 37, 3406 (1988)

17. C. Wetterich, Nucl. Phys. B 302, 668 (1988)

18. R.R. Caldwell, R. Dave, P.J. Steinhardt, Phys. Rev. Lett. 80, 1582 (1998)

19. I. Zlatev, L.M. Wang, P.J. Steinhardt, Phys. Rev. Lett. 82, 896 (1999)

20. R.R. Caldwell, Phys. Lett. B 545, 23 (2002)

21. S. Nojiri, S.D. Odintsov, Phys. Lett. B 562, 147 (2003)

22. Y.H. Wei, Y. Tian, Class. Quantum Gravity 21, 5347 (2004)

23. M.R. Setare, Eur. Phys. J. C 50, 991 (2007)

24. B. Feng, X.L. Wang, X.M. Zhang, Phys. Lett. B 607, 35 (2005)

25. Y.F. Cai et al., Phys. Rep. 493, 1 (2010)

26. A. Sen, JHEP 0207, 065 (2002)
27. T. Padmanabhan, Phys. Rev. D 66, 021301 (2002)

28. M.R. Setare, J. Sadeghi, A.R. Amani, Phys. Lett. B 673, 241 (2009)

29. C. Armendariz-Picon, V.F. Mukhanov, P.J. Steinhardt, Phys. Rev. Lett. 85, 4438 (2000)

30. M.C. Bento, O. Bertolami, A.A. Sen, Phys. Rev. D 66, 043507 (2002)

31. U. Debnath, A. Banerjee, S. Chakraborty, Class. Quantum Gravity 21, 5609 (2004)

32. Z.H. Zhu, Astron. Astrophys. 423, 421 (2004)

33. M.R. Setare, Phys. Lett. B 648, 329 (2007)

34. L. Xu, J. Lu, Y. Wang, Eur. Phys. J. C 72, 1883 (2012)

35. Y. Wang et al., Phys. Rev. D 87, 083503 (2013)

36. H. Saadat, B. Pourhassan, Astrophys. Space Sci. 344, 237 (2013)

37. B. Pourhassan, E.O. Kahya, Results Phys. 4, 101 (2014)

38. B. Pourhassan, E.O. Kahya, Adv. High Energy Phys. 2014, 231452 (2014)

39. E.O. Kahya, B. Pourhassan, Astrophys. Space Sci. 353, 677 (2014)

40. E.O. Kahya, B. Pourhassan, Mod. Phys. Lett. A 30, 1550070 (2015)

41. E.O. Kahya et al., Eur. Phys. J. C 75, 43 (2015)

42. J. Sadeghi et al., Eur. Phys. J. Plus 130, 84 (2015)

43. B. Pourhassan, Can. J. Phys. 94, 659 (2016)

44. J. Sadeghi et al., Int. J. Theor. Phys. 55, 81 (2016)

45. S. Nojiri, S.D. Odintsov, Gen. Relativ. Gravit. 38, 1285 (2006)

46. L. Susskind, J. Math. Phys. 36, 6377 (1995)

47. P. Horava, D. Minic, Phys. Rev. Lett. 85, 1610 (2000)

48. S. Thomas, Phys. Rev. Lett. 89, 081301 (2002)

49. S.D.H. Hsu, Phys. Lett. B 594, 13 (2004)

50. M. Li et al., J. Cosmol. Astropart. Phys. 9, 021 (2013)

51. M. Tavayef, A. Sheykhi, K. Bamba, H. Moradpour, Phys. Lett. B 781, 195 (2018)

52. M. Sharif, S. Saba, Symmetry 11, 92 (2019)

53. S. Ghaffari, H. Moradpour et al., Eur. Phys. J. C 78, 706 (2018)

54. M. Abdollahi Zadeh et al., Eur. Phys. J. C 78, 940 (2018)

55. V. Gujan et al., New Astron. 70, 36 (2019)

56. E. Sadri, Eur. Phys. J. C 79, 762 (2019)

57. Y. Aditya et al., Eur. Phys. J. C 79, 1020 (2019)

58. M. Sharif, S. Saba, Symmetry 11, 92 (2019)

59. B. Mishra et al., Astrophys. Space Sci. 363, 86 (2018)

60. Y. Aditya, D.R.K. Reddy, Int. J. Geom. Methods Mod. Phys. 15, 1850156 (2018)

61. M. Sharif et al., Symmetry 10, 153 (2018)

62. Y. Aditya, D.R.K. Reddy, Astrophys. Space Sci. 363, 207 (2018)

63. D. Vipin Chandra et al., Pramana J. Phys. 93, 78 (2019)

64. M. Korunur, Mod. Phys. Lett. A 34, 1950310 (2019)

65. M.A. Zadeh, A. Sheykh, H. Moradpour, K. Bamba, Preprint. arXiv:1901.05298 (2019)

66. U.Y. Divya Prasanthi, Y. Aditya, Results Phys. 17, 103101 (2020)

67. M. Vijaya Santhi, Y. Sobhan Babu, Eur. Phys. J. C 80, 1198 (2020)

68. M. Vijaya Santhi, Y. Sobhan Babu, New Astron. 89, 101648 (2021)

69. C.B. Collins et al., Gen. Relativ. Gravit. 12, 805 (1980)

70. K.S. Adhav, Int. J. Astron. Astrophys. 1, 204 (2011)

71. M.V. Santhi et al., Can. J. Phys. 95, 179 (2017)

72. M. Tavayef et al., Phys. Lett. B 781, 195 (2018)

73. H. Wei, Commum. Theor. Phys. 56, 972 (2011)

74. Y.D. Xu, Astrophys. Space Sci. 350, 855 (2014)

75. Y.D. Xu, Commun. Theor. Phys 72, 015402 (2020) 